\title{
Entrehojas: Revista de Estudios Hispánicos
}

Volume 4 | Issue 1

Article 6

Date Accepted: April 272014

Date Published: January 012014

\section{Las aportaciones de los exiliados españoles a México y una nota sobre Buñuel}

Jonathan Godínez Páez

University of Texas-Pan American, jgodinez@utpa.edu

Recommended Citation/Citación recomendada

Godínez Páez, Jonathan (2014) "Las aportaciones de los exiliados españoles a México y una nota sobre Buñuel," Entrehojas: Revista de Estudios Hispánicos: Vol. 4 : Iss. 1 , Article 6. 


\title{
Las aportaciones de los exiliados españoles a México y una nota sobre Buñuel
}

\begin{abstract}
Resumen
RESUMEN El presente artículo brinda una mirada panorámica del exilio español a México tras el estallido de la Guerra Civil española en 1936. Se inicia con un recuento histórico de las vidas de los exiliados españoles, desde la llegada de los niños de Morelia y la creación de La Casa de España hasta su eventual adaptación al país que los recibió. Se discuten brevemente las dificultades a las que se enfrentaron los exiliados tras su llegada, para después puntualizar la manera en la que su integración repercutiría en la vida cultural, académica y científica del México de la época. Además de lo anterior se dedica un espacio a Luis Buñuel y sus aportaciones al cine mexicano. Independientemente de que la llegada de Buñuel a México se da por razones distintas a las de los exiliados su estancia en el país coincide con la de otros compatriotas exiliados.

ABSTRACT This article offers a panoramic view of the Spanish exile to Mexico after the outbreak of the Spanish Civil War in 1936. It begins with a look back at the lives of the Spanish exiles; from the arrival of Los niños de Morelia and the creation of La Casa de España up until their eventual adaptation to the country that received them. The difficulties that the exiles faced after their arrival are briefly discussed to later point out how their integration influenced the cultural, academic and scientific Mexico of that time. In addition to what's previously stated, there's a space dedicated to Luis Buñuel and his contributions to the Mexican film industry. Aside from the fact that Bunuel's arrival happens for different reasons from the exiles', his residing in the country coincides with that of other exile countrymen.
\end{abstract}

\section{Keywords/Palabras clave}

exilio, guerra civil, cine mexicano, Lázaro Cárdenas, Universidad Nacional de México 


\section{Introducción}

Tras el estallido de la Guerra Civil en España en 1936 y gracias al apoyo brindado por el presidente Lázaro Cárdenas a la causa republicana, se inicia un éxodo de españoles a México a partir de 1937 con la llegada de los "niños de Morelia". Más tarde en 1938 llegaría a México un grupo pequeño de intelectuales, para quienes se establecería La Casa de España propuesta por don Daniel Cosío Villegas al presidente Lázaro Cárdenas. Sin embargo tras el triunfo franquista en 1939, se inicia un traslado más numeroso de españoles hacia la república mexicana. Provenientes de puertos franceses llegan a Veracruz varias embarcaciones, siendo la más numerosa y conocida la del Sinaia, en la cual se calcula llegaron alrededor de 1599 refugiados (Blanco 17). Desde su llegada los españoles exiliados tuvieron un impacto importante en la sociedad mexicana en distintos y variados ámbitos. A continuación una vista panorámica de las aportaciones, colaboraciones y el legado que dejaron y construyeron los refugiados españoles en distintos sectores de la sociedad mexicana. Iniciando con un recuento histórico del exilio español en México, desde la llegada de los "niños de Morelia" y la fundación de La Casa de España hasta su desenvolvimiento y adaptación en distintos ámbitos de la vida mexicana. El presente trabajo pretende exponer la importancia de la presencia española en la historia, la educación, las artes y la química en México, además de recalcar la importancia de varios personajes de este periodo histórico, entre ellos: Félix Candela, Luis Buñuel, Joan Renau, José Gaos y Antonio Madinaveitia. Como objetivo principal intenta en pleno siglo XXI recordar y puntualizar la importancia que ha tenido el exilio español en la historia y el desarrollo de México.

México nunca se ha caracterizado por ser un país de inmigrantes. Si bien es cierto que desde la conquista ha existido en México una presencia española, ésta nunca ha tenido una importancia numérica en su población. La presencia peninsular en México y particularmente la que llega como consecuencia del estallido de la Guerra Civil en España, lejos de repercutir numéricamente en el país, influye de manera cualitativa en numerosos aspectos de la vida en México, como la educación, la ciencia y las artes (Bonfil 425-35).

\section{Contexto histórico}

A pesar de que el grupo de refugiados que llegó a México no era exclusivamente de intelectuales, sí era un "grupo que, por sus intereses, no estaba destinado a emigrar, y que no lo hubiera hecho motu propio si no hubiera sido por las circunstancias violentas que lo obligaron a desterrarse por la fuerza" (Bonfil 443). Se calcula que del total de refugiados que llegaron a México, el 28\% estaba compuesto por intelectuales, del porcentaje restante, un $72 \%$, poco se ha estudiado (Pla Brugat 182). Lo que sí se sabe es que la mayoría de refugiados que llegó a México se asentó en la capital del país "ahí habrían de vivir casi tres 
cuartas partes de ellos" (Pla Brugat 167), es por eso que de la Ciudad de México es de donde se tiene más registro de sus contribuciones y de su desenvolvimiento en la vida mexicana.

En 1937 llega el primer grupo de refugiados españoles a México, históricamente conocidos como los "niños de Morelia". Este grupo de aproximadamente 500 niños (existen diferentes cifras) llega a Morelia como consecuencia del estallido de la Guerra Civil en España en julio de 1936 (Pla Brugat 162). Tras el inicio del conflicto armado un número importante de familias españolas republicanas deciden enviar a sus hijos a México con la idea de que el conflicto armando duraría poco tiempo y además teniendo en mente la idea de que los republicanos se alzarían con la victoria. Por razones históricas que ahora conocemos, como la prolongación de la Guerra Civil y la derrota republicana, los "niños de Morelia" permanecerían, en su mayoría, su vida entera en México. A su llegada a México los "niños de Morelia" fueron muy bien recibidos por la gente mexicana, además de que el gobierno y personalmente el presidente Lázaro Cárdenas siempre estuvo en contacto con esta generación de niños que permanecieron tras su llegada en la Escuela España-México que funcionaba como un internado. Con el paso de los años el internado dejo de funcionar, los niños crecieron y se asentaron a la vida diaria del país que los acogió. Sin embargo como todo exiliado la vida no fue fácil, la separación de sus padres, sus cortas edades y la lejanía de su país, fueron obstáculos que poco a poco tuvieron que superar. En su mayoría como lo comparte Dolores Pla Brugat (especialista en el tema del exilio español) en una entrevista para el podcast Mirar a Fondo MéxicoBicentenario, la mayoría de ellos se volvieron personas productivas para el país.

Para 1938, con la llegada de un grupo de intelectuales, entre los que se encontraba el rector de la Universidad de Madrid José Gaos, se establece ese año La Casa de España en la Ciudad de México. La Casa de España surge con la idea de dar un espacio a los intelectuales españoles de continuar su labor intelectual, científica y cultural mientras se vivía en España la Guerra Civil. Es importante puntualizar que La Casa de España se convertiría en un "refugio" para un gran número de intelectuales españoles. Bajo la presidencia de Alfonso Reyes, esta institución se encargó de posicionar y distribuir a estos intelectuales en distintos centros académicos: la Universidad Nacional, el Instituto Politécnico Nacional (IPN), el Fondo de Cultura Económica y muchos otros centros de estudio alrededor del país (Hernández 131). El mismo Alfonso Reyes en un artículo de 1939 definiría la principal función de La Casa de España en México: "Its function is to invite to Mexico and make available to all the culture centers of the coutry the Spanish scholars, professors and writers of note who thereby constitute its staff of "Miembros Residentes"' (415).

Con la llegada en 1939 de un número mayor de españoles que se integraron a La Casa de España y de muchos otros mexicanos que hicieron lo 
mismo - "Mexican intellectual leaders, who are thus placed in contact and collaborartion with Spaniards" (Reyes 415) - cambia su nombre en 1940 a El Colegio de México. Posteriormente en 1943 se funda el Centro de Estudios Históricos bajo la dirección de Silvio Zavala, inspirado en el Centro de Estudios Históricos de Madrid. El mismo año se inauguraría el Centro de Estudios Sociales y años más tarde el Centro de Estudios Filológicos (Hernández 132-33).

\section{La Universidad Nacional de México, el Instituto Politécnico Nacional (IPN) y el Fondo de Cultura Económica}

Además de contribuir con la expansión y desarrollo del Colegio de México, los intelectuales españoles tuvieron un impacto en otras instituciones educativas. Tras su llegada estos profesores, filósofos y científicos, trajeron consigo nuevas ideas y teorías que vinieron a enriquecer a las instituciones que los recibieron. Mientras La Casa de España se expandía y cambiaba de nombre, la Universidad Nacional vivía un momento en su historia de reorganización tras lograr su autonomía en 1929. Así la Facultad de Filosofía y Letras abrió espacio para más profesores. Llegaron a la Universidad historiadores, antropólogos y filósofos, que ayudaron a organizar nuevos programas de posgrado. Entre ellos José Gaos, Adolfo Sánchez Vázquez y Carlos Boch García.

Al llegar este vasto grupo de intelectuales ayudaron a formar el Centro de Estudios Filosóficos y el Instituto de Investigaciones Históricas. Dentro de la Facultad de Derecho ayudaron a crear el Instituto de Investigaciones Jurídicas y a desarrollar el programa de doctorado en derecho. Hoy en día en la página de presentación de la Facultad de Filosofía y Letras de la Universidad Nacional Autónoma de México (UNAM) filos.unam.mx se puede leer lo siguiente:

Diez años después, la Facultad, la Universidad y el país entero recibió una fuerza de enorme renovación intelectual con los maestros del exilio español, quienes después de haber defendido con las letras y con las armas la república española establecida democráticamente hace 75 años, se vieron obligados a dejar su patria para realizar en nuestro país y particularmente en nuestra Universidad los ideales humanistas en el campo de las humanidades, las artes y las ciencias.

Dentro de la universidad, además colaboraron en la publicación de distintas revistas como la Revista de Filosofía y Letras fundada en 1941 y la Revista de la Universidad. De la Revista de Filosofía y Letras salieron cincuenta y cuatro volúmenes siendo una gran fuente del pensamiento humanístico e intelectual de la década de 1940 (Hernández 136).

En el Instituto Politécnico Nacional al igual que en la Universidad Nacional, los refugiados españoles colaboraron en la vida académica, entre ellos Ignacio Bolívar y Urrutia que trabajó en la Escuela Nacional de Ciencias 
Biológicas creada en 1934 (Hernández 139). Llegaron al IPN cerca de 60 investigadores formados en España y en varias universidades europeas importantes. No sólo impartieron cátedra y realizaron trabajos de investigación sino que además colaboraron en el desarrollo de nuevos programas de posgrado como el de Bioquímica y el de Ingeniero Bioquímico. Colaboraron en la creación del Laboratorio de Fisiología General y Vegetal de la Escuela Nacional de Ciencias Biológicas, además de participar en la fundación de revistas académicas. Entre otras de sus contribuciones se encuentra la creación de laboratorios especializados como el de Paleoecología en Petróleos Mexicanos. De acuerdo con la revista El Cronista del Instituto Politécnico Nacional, en dos mil cinco junto con la Universidad Autónoma de México (UNAM) esta institución recibió la medalla de honor de parte de la Universidad Complutense de Madrid "como agradecimiento por haber acogido a los académicos españoles exiliados durante la guerra civil española" (27).

Por su parte en el Fondo de Cultura Económica, fueron muchos los españoles que tras su llegada en mayor número en 1939 colaboraron con dicha institución. Con su llegada el Fondo de Cultura Económica dio la bienvenida a nuevos editores, traductores, formadores y demás personal capacitado que ayudó enormemente en la expansión de la institución. Para 1939 aparecen los primeros libros de sociología e historia, en una institución donde en un principio y tras su fundación se tenía la idea de sólo editar libros sobre economía. Gracias a las traducciones y el trabajo de edición de distintos exiliados españoles se pudo ampliar enormemente el catálogo de obras que ofrecía dicha institución. Entre los intelectuales que trabajaron y contribuyeron al desarrollo del Fondo de Cultura Económica se encuentran: José Gaos, León Felipe y Enrique Díez Canedo; hombres que encontraron en México un lugar donde seguir desarrollando su intelecto y continuar con sus vidas laborales.

\section{Aportaciones en la Bibliografía Mexicana}

Los españoles refugiados llegaron a México a aportar y si bien en un principio su idea era la de regresar a España, tras el posible triunfo republicano, esto no impidió que se involucraran de lleno a la vida mexicana. Otra colaboración que es importante mencionar es la de la creación de la Bibliotheca Scriptorum Graecorum et Romanorum Mexicana. La creación de esta biblioteca encargada de traducir al castellano las obras de la Grecia clásica y del Imperio Romano se le debe a Agustín Millares Carlo, académico y paleógrafo. Dentro de la biblioteca desde la aparición de los primeros volúmenes en 1944 y hasta 1955 las traducciones fueron hechas en su mayoría por españoles; entre ellos: Juan David García Bacca, José María Gallegos Rocafull y por supuesto Agustín Millares Carlo. Aunado al gran interés por manuscritos latinos y novohispanos, Agustín Millares Carlo, "fue un profundo conocedor de la bibliografía teórica, universal, 
nacional especializada y otro tanto de la archivología histórica de Indias y de los acervos documentales latinoamericanos" (Perales 6). Debido a su gran conocimiento y capacidad se le encomendó realizar una nueva edición de la Bibliografía Mexicana del Siglo XVI de don Joaquín García Icazbalceta, con una extensión de 362 ejemplares publicada por el Fondo de Cultura Económica en 1954, una aportación de suma importancia (Perales 6-7).

Entre otros personajes importantes que colaboraron en la bibliografía mexicana se encuentra también Alfonso Ayensa quien tras su llegada a México en 1952 proveniente de Francia "fue director y editor de la Bibliografía para investigadores de problemas económicos, planificación y desarrollo de las diversas ramas de la industria" (Perales 9). Además dirigió la Bibliografía Industrial de México (18 volúmenes), el Repertorio bibliográfico de ciencia y tecnología CONACYT y fue asesor del Banco de México (Perales 9). Se le considera el primer documentista mexicano por el gran trabajo recopilatorio que hizo del Banco de México y sus trabajadores.

Importante es mencionar que si bien los exiliados españoles lograron asentarse en la vida del país que los recibió y brindó apoyo esto no fue fácil. Aunado a la nostalgia se encontrarían con un sentimiento anti-español que tras la conquista y la revolución era inminente en la población mexicana. Se encontrarían con un país que si bien les abría las puertas, también nulificaba su participación en cuestiones políticas. Muchos intelectuales activamente involucrados en la política de su país, a su llegada a México tuvieron, en gran parte, que desprenderse de ese rol ya que la constitución mexicana restringía la participación de cualquier extranjero en cuestiones políticas (Faber 222). Sin embargo, estas situaciones adversas a las que se enfrentarían los refugiados españoles, no iban a impedir su activa participación en la sociedad mexicana, especialmente en el plano intelectual. Al adentrarse en la sociedad mexicana lograrían también cambiar la percepción que se tenía en torno a sus compatriotas que denominaban "gachupines" y que habían llegado a México en distintas épocas y por razones distintas a las de los refugiados.

Debemos además mencionar que si bien México los recibió y les brindó un lugar donde continuar su vida lejos de la guerra, los españoles refugiados contaron con la protección del gobierno republicano por medio de dos organismos de ayuda que les brindaron un sinnúmero de apoyos tras su llegada a México. Se les brindó desde un alberge donde dormir, hasta préstamos para iniciar negocios, "no sé cómo habríamos sobrevivido los primeros años en México sin ellas" dice Blanco Aguinaga (21). Estas agencias de ayuda eran el SERE (Servicio de Evacuación de Republicanos Españoles) y el JARE (Junta de Ayuda a los Republicanos Españoles). De acuerdo con Pla Brugat estas agencias ayudaron y apoyaron a un número aproximado de 16,000 personas (163). 


\section{Los Colegios del Exilio}

Los españoles refugiados, no sólo colaboraron en la expansión y desarrollo de las instituciones académicas mexicanas, sino que también crearon centros educativos. El propósito de estos colegios era en un principio, permitir a los hijos de los españoles republicanos mantener su identidad española y por otro lado dar empleo a los muchos maestros que habían llegado de España y a los cuales resultaba complicado posicionar por medio de La Casa de España. Entre estos colegios se encuentra el Instituto Luis Vives, una institución que gracias al nivel académico contó con el apoyo de intelectuales mexicanos y el mismo presidente Lázaro Cárdenas. En el Instituto Luis Vives, se reunió a un número considerable de exiliados, que colaboraron activamente en el desarrollo del colegio (Monedero 12). Hoy en día de acuerdo con el portal de la institución, el $80 \%$ de los estudiantes son mexicanos y solo el $20 \%$ son hijos o nietos de los antiguos fundadores.

Después de la creación del Instituto Vives, se creó la Academia Hispano Mexicana. Esta institución en un principio fue un colegio que contaba con educación secundaria y preparatoria para después incluir la educación primaria. La institución se fundó gracias al apoyo de fondos otorgados por el SERE y algunos accionistas mexicanos. "Entre la emigración se decía que la academia representaba 'la aristocracia de la democracia' y se tachaba al Vives de ser un semillero de comunistas alborotadores" (Monedero 4). A pesar de las diferencias políticas e ideológicas en distintos sectores de la población republicana en México, los intelectuales refugiados siempre trataron de mantener la solidaridad entre su gente (Blanco 18).

En 1941 se funda El Colegio Madrid que actualmente sigue con su labor académica en la Ciudad de México. Fundada con la ayuda de la Junta de Ayuda a los Republicanos Españoles (JARE). De esta institución de alto nivel académico salieron generaciones de estudiantes reconocidas por la Universidad Autónoma de México y el Instituto Politécnico Nacional (Monedero 5). De acuerdo con la institución, su visión ha sido hasta ahora la implantación de una enseñanza basada en un modelo pedagógico de la institución libre de enseñanza creada por el filósofo y educador Francisco Giner de los Ríos. Una institución donde el estudiante tenga un lugar para la crítica y la reflexión. Actualmente además el bachillerato que se ofrece está incorporado a la Universidad Nacional Autónoma de México.

Otras instituciones educativas que se fundaron gracias a la presencia de los refugiados españoles son las siguientes: la Academia Hispano Mexicana, el Colegio Cervantes de Torreón (activo), el Instituto Escuela Cervantes en Tamaulipas (activo) y el Colegio Hispano-Mexicano de Torreón (activo), entre otros. En un principio estos colegios se fundaron con la idea de que los hijos de los refugiados no perdieran su identidad española e ideología republicana, ahora 
muchos de ellos siguen operando con un alumnado y un personal que es mayoritariamente mexicano.

\section{Félix Candela y la arquitectura}

Las aportaciones de los exiliados españoles a México no se limitan exclusivamente a las áreas de educación y humanidades. Fuera de los salones de clase y de las instituciones académicas tuvieron además, aportaciones en otros campos como en la arquitectura. Entre los arquitectos que ingresaron a México tras la derrota republicana en la Guerra Civil se encontraba Félix Candela, un joven que había estudiado en la escuela de Arquitectura de Madrid. Admirador de las estructuras laminares europeas, Candela, tras diez años de permanecer en México, funda junto con sus hermanos y otros arquitectos mexicanos, una empresa llamada Cubiertas Ala (Del Cueto 31-33). Gracias a su idea de construir "cascarones de concreto", Candela logró construir los primeros prototipos de paraguas. Desde su aparición fue este tipo de cubierta el que se convirtió en la preferida por arquitectos y empresarios para cubrir grandes superficies. Para ilustrar mejor esta idea del término paraguas y cascarones de concreto basta con mirar una foto del Palacio de los Deportes o de la Bolsa de Valores México.

Estas ideas del paraguas y el cascarón de concreto son sellos del trabajo del arquitecto Candela que se puede observar no sólo en las dos construcciones antes mencionadas sino además en varias iglesias, mercados, restaurantes y la Planta Embotelladora de Bacardí. Esta innovación arquitectónica resultó muy fructífera ya que el costo de construcción era relativamente económico. El trabajo de Candela fue y sigue siendo un sello en la arquitectura mexicana ya que en otros países estas construcciones (cascarones) no cumplían con las normas mínimas de seguridad debido a que las laminas de hormigón que se empleaban eran demasiado delgadas y representaban una amenaza a la seguridad de la construcción (Del Cueto 35).

Además de las importantes e innovadoras ideas arquitectónicas de Candela y la colaboración de arquitectos mexicanos en su trabajo, México lo recibió en una época de crecimiento económico que se vio reflejado en la industria de la construcción. En las siguientes palabras el arquitecto Candela sintetiza su trabajo y aportación a la arquitectura mexicana y a la industria de la construcción en general:

Mi mayor satisfacción no estriba en haber ejecutado ciertas estructuras espectaculares -aunque confieso que he disfrutado mucho haciéndolas-, sino en haber contribuido, siquiera sea en forma mínima, a aliviar el ingente problema de cubrir económicamente espacios habitables, demostrando que la construcción de cascarones no constituye una hazaña extraordinaria que inmortalice a sus autores, sino un procedimiento constructivo sencillo y flexible. (citado en Del Cueto 40) 
Es importante tener en cuenta que su arquitectura es parte de la panorámica e historia de la Ciudad de México.

\section{Luis Buñuel y el cine mexicano}

Luis Buñuel es otro exiliado español que tuvo grandes aportaciones a la sociedad mexicana especialmente en el ámbito cinematográfico. El director Luis Buñuel no llega a México en los primeros años del exilio (1937-1939) sino que llega años después tras permanecer en Estados Unidos por varios años (1938-1946) tras el triunfo del franquismo en España. La llegada a México de Buñuel es de suma importancia para la historia del cine mexicano como lo recalca en repetidas ocasiones Acevedo-Muños en su libro Buñuel and Mexico: The Crisis of National Cinema. Su llegada es particularmente importante ya que el cine mexicano se encontraba en una crisis a mediados de los años 40, las películas de Emilio "el Indio" Fernández y Gabriel Figueroa habían tenido ya su mejor momento (sobre todo en el extranjero), los temas en el cine, los escenarios, los roles de los actores y las locaciones se volvieron repetitivos. Por otra parte el gobierno mexicano sólo brindaba apoyo a las películas que recurrieran a temas meramente mexicanos, tales como la revolución, para que tuvieran exposición en los festivales internacionales de cine. La crítica internacional había perdido el interés por el cine mexicano y a su vez el cine mexicano había perdido reconocimiento.

Con la llegada de Buñuel y especialmente con su película Los olvidados con la que ganó el premio como mejor director en el Festival de Cannes (Faber 232), Buñuel le dio voz nuevamente al cine mexicano. Durante su estancia en México el director español se encontraría, como la mayoría de los exiliados con dificultades para adaptarse y sobresalir. Buñuel tendría que enfrentarse con las distintas limitantes del cine mexicano de la época, en especial con la falta de presupuesto y equipo para los rodajes. Sin embargo pese a las limitantes Buñuel produjo 17 de sus 32 filmes en México. Del trabajo que realizó el director español se pueden recalcar varios filmes pero una mención especial merece el filme Los olvidados de 1950, no sólo por el reconocimiento que obtuvo de la crítica internacional sino por el tema que toca dicha película, la pobreza, la modernización y la fallida estrategia gubernamental para resolver los problemas sociales del país. Los olvidados fue vista de manera negativa, no simplemente por el tema que tocaba, sino porque el filme era dirigido por un extranjero, hubo algunas personas que incluso pidieron la expulsión del país del director español (Faber 232). Los olvidados presenta una parte de la sociedad sumida en la pobreza y el abandono, cobijada por la corrupción, el olvido, la delincuencia y la pérdida de la esperanza por un futuro mejor, todo esto en medio de la modernización de la Ciudad de México (Rubia 396-97).

Los problemas que el filme Los olvidados ocasionó a Buñuel es un reflejo de las vivencias de los exiliados en México. Por un lado los españoles fueron 
invitados a formar parte de la sociedad mexicana, pero por otro estaban siempre frente a limitaciones que les imponía el hecho de ser extranjeros. El trabajo de Buñuel es difícil de resumir en unas cuantas líneas, sin embargo entre sus aportaciones al cine están el hecho de tratar nuevos temas en el cine mexicano, parodiar los temas recurrentes de los melodramas de la época, alejarse de los personajes clásicos como el charro, el revolucionario, el macho mexicano y tal como en Los olvidados alejarse también de la panorámica de las montañas y los pueblos tan característicos del cine de la época, para pasar a la modernidad de la ciudad. Buñuel además ayudó a la transición del cine mexicano con sus producciones, pasando del cine clásico al nuevo cine de finales de los años 60. La sumisión de México a la modernidad representa lo anti-clásico en el cine mexicano, representa una evolución (Acevedo 72). Buñuel revolucionó el cine una década antes tratando temas distintos a los del cine clásico y siendo la contraparte de los filmes de Emilio Fernández, renovando el estilo, la ideología, la imagen y en general la dirección del viejo cine (Acevedo 151). Entre las películas más destacadas de su periodo en México se pueden mencionar las siguientes: Los Olvidados, El bruto, Él, Ensayo de un crimen, Subida al Cielo, La ilusión viaja en tranvía, El río y la muerte y Una mujer sin amor, entre otras. Hoy en día es imprescindible el estudio de Buñuel para comprender la historia y el desarrollo del cine en México.

\section{Aportaciones en el arte}

Los artistas españoles refugiados tuvieron también repercusiones en la vida mexicana. Desde su viaje estos artistas realizaron exposiciones de dibujo a bordo del Sinaia. A su llegada a México como varios más de sus compatriotas los artistas encontraron trabajo como maestros de dibujo y arte en colegios como el Instituto Luis Vives y el Colegio Madrid. Llegaron a México desde futuros artistas como el escultor Julián Martínez Soto en 1937, perteneciente al grupo de los "niños de Morelia" hasta críticos de arte como Moreno Villa o Juan de la Encina ya activos desde su llegada (Cabañas 59). En la Ciudad de México los artistas además abrieron un gran número de cafés y galerías para sus tertulias y exposiciones, además crearon la institución, el Ateneo Español, fundado en 1949, un sitio que se convirtió en un centro cultural muy importante. En este centro se dieron cita tanto pintores como escultores, escritores, fotógrafos, y demás artistas (Cabañas 62). Hoy en día el Ateneo Español de México A.C., continúa con sus labores ofreciendo espacios para el estudio del exilio español en México, además de contar con biblioteca y salas de exposición.

Joan Renau es otro artista importante dentro del exilio en México, fundador de un taller de cartelismo llamado Estudio Imagen-Publicidad Plástica. Por medio de este taller Renau realizó un gran número de carteles para las productoras más importantes de aquellos tiempos entre ellas: Arofilms, 
Producciones Calderón, Filmex, Mier y Brooks y Ultramar Films. Realizaría carteles también para campañas del PRI y de la ONU. Por otro lado la Agencia Hermanos Mayo (fundada al comienzo de la Guerra Civil y continuada en México) realizó un trabajo importante de fotografía para periódicos y publicaciones mexicanas como: El Popular, La Prensa, Mañana, El Diario de México y muchos más (Cabañas 63-66). Los hermanos Mayo cedieron su trabajo en 1994 al Archivo General de la Nación en México y en 2007 fueron declarados Premio Nacional de Periodismo por su trayectoria periodística. Se debe mencionar además que si bien los artistas del exilio fueron lentamente asentándose en la vida mexicana, este asentamiento no fue nada fácil. Mientras los artistas españoles llegaban con ideas e influencias europeas, en México el muralismo vivía un auge importante. Debido a este choque de ideas algunas veces se les acusó a los artistas españoles de intentar en contra de la corriente social y objetivista que reflejaba el muralismo (Cabañas 66).

\section{Aportaciones en la química}

En la química los exiliados también tuvieron aportaciones importantes. Llegaron a México y se interesaron por los problemas del país, incluso en palabras del químico Antonio Madinaveitia el medio científico era muy parecido al español así que les fue fácil adaptarse. En uno de los proyectos de mayor trascendencia en que tuvieron presencia los químicos del exilio fue en Sosa Texcoco, empresa mexicana que explotaba minas de salmueras alcalinas naturales. Esta empresa pretendía explotar el lago de Texcoco con fines agrícolas. Madinaveitia propuso un proceso exitoso que permitió comercializar el carbonato y la sal al obtenerlos por separado. Ayudó además a la fundación del Instituto de Química de la Universidad Nacional, en donde participó en la formación de personalidades de la química mexicana de mediados del siglo XX, como Octavio Mancera, Jesús Romo Armería, y Humberto Estrada. Realizó estudios sobre el pulque, los aguarrases mexicanos y el mercurio (Capella et al. 61-63). Cabe mencionar también a José Giral quien además de dirigir más de 150 tesis en química, formó en México el primer grupo de investigadores de química de alimentos.

Por su parte el doctor Francisco Giral González, hijo de José Giral, creó el posgrado en ciencias químicas farmacéuticas en la Universidad Nacional, además de ser considerado fundamental en el desarrollo de las ciencias farmacéuticas en México. José Ignacio Bolívar Goyanes, otro químico que llegó de joven a México y que estudiara en Harvard en la Facultad de Medicina, implementó técnicas para producir comercialmente por primera vez en México sueros contra la difteria y el tétenos (Capella et al. 66-67). Además los químicos exiliados fundaron laboratorios farmacéuticos e industrias químico farmacéuticas. 


\section{Conclusiones}

Son innegables y numerosas las aportaciones que tuvo la llegada de los exiliados españoles a México. Es difícil encontrar un espacio en la vida nacional mexicana donde no haya tenido un impacto, una colaboración o presencia algún exiliado. Aquí hemos discutido panorámicamente las aportaciones en la educación, la bibliografía, la arquitectura, el cine, el arte y la química. Es sin embargo importante mencionar que las aportaciones hoy reconocidas de los refugiados españoles no se limitan a estas áreas. Sobre este tema se podría realizar un trabajo mucho más extenso ya que las aportaciones fueron muchas y muy variadas. Entre las no mencionadas están por ejemplo las de Álvaro Custodio en teatro, en televisión de Luis de Llano Palmer, en filosofía de Adolfo Sánchez Vázquez y José Gaos, en poesía de Luis Cernuda y Max Aub; en fin un extenso y variado número de aportaciones en distintas áreas.

La llegada de los españoles refugiados coincidió con una época en México de desarrollo en distintas áreas como la economía, la educación y la cultura. Los exilados llegaron en un tiempo muy especial, por ejemplo, a pocos años de la fundación del IPN (1936), a poco tiempo de la creación del Fondo de Cultura Económica (1934), a una década de que la Universidad Nacional lograra su autonomía. Llegaron en un momento de renovación y modernización del país, es decir en una época casi "ideal" para que los intelectuales españoles continuaran su desarrollo laboral y académico mientras contribuían en el aspecto intelectual, principalmente, al desarrollo del país que los recibió. Independientemente de como se sintieron tras su llegada a México ya sea como desterrados, transterrados o mexicanizados, sus colaboraciones son de un valor incalculable. Sus aportaciones a la historia intelectual y social de México no deben de ser olvidadas pues todavía quedan las huellas y semillas de sus contribuciones.

\section{Obras Citadas}

Acevedo Muñoz, Ernesto R. Buñuel and México: The Crisis of National Cinema. London: University of California Press, 2003. Impreso.

Bonfil Batalla, Guillermo. Simbiosis de culturas: los inmigrantes y su cultura en México. México D.F: Fondo de Cultura Económica, 1993. Impreso.

Blanco Aguinaga, Carlos. "A Modo de prólogo: Sobre la especificidad del exilio español en México." Ensayos sobre la literatura del exilio español. México: El Colegio de México, 2006. 11-26. Impreso.

Cabañas Bravo, Miguel. "Los artistas españoles del éxodo y el llanto bajo el techo azteca." ARBOR Ciencia, Pensamiento y Cultura 180.735 (2009): 57-74. Impreso. 
Capella, Santiago, et al. "La huella en México de los químicos del exilio español de 1939." Científicos y humanistas del exilio español en México. México: Academia Mexicana de Ciencias, 2006. 155-172. Impreso.

Del Cueto Ruiz Fuenes, Juan Ignacio. "Félix Candela, el mago de los cascarones de concreto." Arquine, Revista Internacional de Arquitectura 1.2 (1997): 31-40. Impreso.

Faber, Sebastiaan. "Between Cernuda's Paradise and Buñuel's Hell: Mexico through Spanish Exiles' Eyes." Bulletin of Spanish Studies: Hispanic Studies and Researches on Spain, Portugal, and Latin America. 80.2 (2003): 219-39. Impreso.

Hernández de León-Portilla, Ascensión. "Revolución Mexicana y exilio español: tesoro, símbolo, legado." Cuadernos Americanos 4.134 (2010): 125-56. Impreso.

Monedero López, Enrique. "Los colegios del exilio.” Cuadernos de la fundación Españoles en el Mundo 1.16 (1996): 1-11. Impreso.

Pla Brugat, Dolores. Entrevista por Gabriel Arámbula "El exilio español en México.” Mirar a Fondo-México Bicentenario, 2010. Web. 1 Abr. 2012.

---. “La presencia española en México, 1930-1990. Caracterización e historiografía.” Migraciones y Exilios 1.2 (2001): 157-88. Impreso.

Perales Ojeda, Alicia. "La aportación del exilio español a la bibliografía nacional de México." Departamento de Bibliotecología Facultad de Filosofía y Letras UNAM. n.d. 1-10. Web. 30 Nov. 2011.

Reyes, Alfonso. “The 'Casa de España en México'.” Books Abroad 13.4 (1939): 414-17. Impreso.

Rubia Barcia, J. 'Luis Buñuel's 'Los Olvidados'." Quartely of Film Radio and Television 7.4 (1953): 392-401. Impreso. 